\title{
Optimisation du NPK et urée basée sur les informations climatiques pour accroitre la production du sorgho en zones soudano-sahéliennes du Sénégal
}

\author{
Komla Kyky GANYO1,2,3*, Bertrand MULLER ${ }^{1,4}$, Espoir Koudjo GAGL01,2, Aliou GUISSÉ2 ${ }^{2}$ Ndiaga CISSÉ1 \\ et Myriam ADAM4,5,6 \\ ${ }^{1}$ Centre d'Étude Régional pour l'Amélioration de l'Adaptation à la Sécheresse (CERAAS), BP 3320, Route de Khombole, \\ Thiès, Sénégal \\ 2 Département de Biologie Végétale, Université Cheikh Anta Diop de Dakar (UCAD), BP 5005, Dakar, Sénégal \\ ${ }^{3}$ Institut Togolais de Recherche Agronomique (ITRA), BP 1163, Lomé, Togo \\ ${ }^{4}$ Centre de Coopération Internationale en Recherche Agronomique pour le Développement (CIRAD), UMR AGAP/PAM, \\ 34090 Montpellier France \\ 5 Institut de l'Environnement et de la Recherche Agricole (INERA), 01BP 910, Bobo Dioulasso, Burkina Faso \\ ${ }^{6}$ International Crops Research Institute for Semi-Arid Tropics (ICRISAT), BP 320, Bamako, Mali \\ *Auteur correspondant : desireganyo@gmail.com
}

Original submitted in on 10 th August 2018. Published online at www.m.elewa.org on $30^{\text {th }}$ November 2018 https://dx.doi.org/10.4314/iab.v131i1.5

\section{RÉSUMÉ}

Objectif : L'objectif est d'étudier les réponses de variétés de sorgho à des apports contrastés de NPK et urée qui pourraient être dictés par les informations météorologiques.

Méthodologie et résultats : Des essais en split plot avec quatre répétitions ont été conduits à Nioro du Rip et Sinthiou Malème (Sénégal) sur sols sableux en conditions pluviales. Quatre variétés (Fadda, Faourou, Soumalemba et Soumba) ont été soumises à cinq modalités de fertilisation : T1 $=$ pas d'engrais ; T2 $=150$ $\mathrm{kg} / \mathrm{ha}$ de NPK (15-15-15) à la levée $+50 \mathrm{~kg} / \mathrm{ha}$ d'urée $(46 \%)$ au tallage $+50 \mathrm{~kg} / \mathrm{ha}$ d'urée à la montaison (recommandation au Sénégal pour le sorgho) ; T3 = moitié dose de T2 appliquée aux mêmes stades; T4 = 150 $\mathrm{kg} / \mathrm{ha}$ de NPK $+50 \mathrm{~kg} / \mathrm{ha}$ d'urée à la montaison $+50 \mathrm{~kg} / \mathrm{ha}$ d'urée à l'épiaison (T2 décalé) et T5 = moitié dose T4 apportée aux mêmes stades. La fertilisation tardive a permis aux plantes stressées, de recouvrir la croissance telle si les apports étaient faits tôt dans la saison. Les rendements ont varié suivant les localités. L'apport décalé de la dose recommandée (T4) a été plus bénéfique que la moitié dose décalée (T5). Les variétés ont montré des réponses différentes les unes des autres, avec Soumalemba qui a mieux réagi aux apports tardifs.

Conclusion et application des résultats : II ressort de cette étude que la fertilisation tardive a été bénéfique aussi bien pour le grain que la biomasse. Elle reste une alternative à la gestion de fertilisation telle que recommandée ou pratiquée par les paysans en cas de début de saison de pluie non favorable surtout dans des pays agropastoraux comme le Sénégal. Les structures d'appui technique aux paysans devront intégrer les prévisions ou observations pluviométriques dans les recommandations de fertilisation afin d'optimiser l'efficience des engrais et la productivité du sorgho.

Mots clés : NPK-urée, optimisation, variabilité pluviométrique, sorgho, Sénégal 

accroitre la production du sorgho en zones soudano-sahéliennes du Sénégal

\section{ABSTRACT \\ Optimizing NPK and urea based on weather forecast to increase sorghum production in Sudano- Sahelian zones in Senegal}

Objective: Rainfall variability and soil nutrients deficiency accentuated by climate change affects sorghum production in Senegal. This work aims to study the responses of sorghum (Sorghum bicolor L. Moench) to contrasting application of NPK and urea that could be based on weather forecasts.

Methodology and results: A split-plot experiment with four replications was carried out at "Nioro du Rip" and "Sinthiou Malème" (Senegal) on sandy soils in rainfed conditions. Four sorghum varieties (Fadda, Faourou, Soumalemba et Soumba) were submitted to five fertilizer treatments defined as T1 $=$ no fertilizer; $\mathrm{T} 2=150$ $\mathrm{kg} / \mathrm{ha}$ of NPK (15-15-15) at emergence $+50 \mathrm{~kg} / \mathrm{ha}$ of urea (46\%) at tillering $+50 \mathrm{Kg} / \mathrm{ha}$ of urea at stem extension (recommended practice in Senegal for sorghum); $\mathrm{T} 3=$ half rate of T2 applied at the same stages; T4 $=150 \mathrm{~kg} / \mathrm{ha}$ of NPK $+50 \mathrm{~kg} / \mathrm{ha}$ of urea at stem extension $+50 \mathrm{~kg} / \mathrm{ha}$ of urea at heading (delay T2) and T5 = half rate of T4 applied at the same stages. Late fertilization allowed plants stressed to recover growth as if fertilizer is applied early in-season. Yield varied according to locations. The application of full dose late inseason (T4) was more beneficial to late application of half rate (T5). Varieties responded differently to fertilization with Soumalemba wich responded better to late application.

Conclusion and application of results: It appears from this study that late fertilization has been beneficial for both grain and biomass. It remains an alternative to fertilization management as recommended or practiced by farmers in the event of an unfavorable rain season, especially in agro-pastoral countries like Senegal. Technical support structures for farmers should integrate rainfall forecasts or observations into fertilization recommendations in order to optimize fertilizer efficiency and sorghum productivity.

Keywords: NPK-urea, optimizing, rainfall variability, sorghum, Senegal

\section{INTRODUCTION}

Le sorgho (Sorghum bicolor (L.) Moench) ) est un des aliments de base de millions de personnes en Afrique (Gueye et al., 2016). Cependant, l'agriculture en générale et la culture du sorgho en particulier en zones Soudano-Sahéliennes d'Afrique sont confrontées à un certain nombre de contraintes parmi lesquelles la faible fertilité des sols (déficiences en nutriments des sols) (Vanlauwe et al., 2010) et la non utilisation ou faible taux d'utilisation d'intrants agricoles (engrais, variétés améliorées...) (Gerstenmier et Choho, 2015). A cela, s'ajoutent les variabilités inter et intra-annuelles de la pluviométrie (Hansen, 2002). En effet, le climat en Afrique est varié et variant. II est varié parce qu'il va des régimes équatoriaux humides, tropicaux arides et à des climats subtropicaux de type méditerranéen, et variant parce que tous ces climats présentent des degrés de variabilité temporelle différents, en particulier en ce qui concerne les pluies (Hulme et al., 2001). Selon IPCC (2007), le changement climatique résultant de l'augmentation des gaz à effet de serre, va accentuer ces variabilités pluviométriques (début et fin des saisons de pluie mais aussi la distribution des pluies) et les évènements extrêmes telles les sécheresses, les inondations et augmentations de la température dans cette partie du monde. L'Afrique subsaharienne est connue pour être particulièrement vulnérable au changement du climat en raison de la combinaison des niveaux naturellement élevés de variabilité climatique, de systèmes agricoles encore dépendant de la pluviométrie et une capacité économique limitée (Challinor et al., 2007; Roudier et al., 2011). Par ailleurs, le changement climatique ajoute du stress et de l'incertitude à la production agricole en Afrique, où de nombreuses régions sont déjà vulnérables à la variabilité climatique. La production végétale dans ces régions devient de plus en plus risquée (Slingo et al., 2005). En effet, percevant les risques liés à la variabilité et au changement climatique, les paysans limitent leurs investissements dans la production des cultures, préférant mettre peu ou pas du tout d'engrais, utiliser des variétés locales afin de maintenir un niveau minimum de production (Amadou et al., 1999; Ingram et al., 2002). Cependant, avec l'explosion 
démographique et une demande alimentaire sans cesse croissante, il y a un réel besoin d'augmenter la productivité des cultures tout en minimisant les pertes et maintenir la résilience. Parmi les nombreuses options techniques possibles (la maîtrise d'eau, les associations culturales, la micro fertilisation, par exemple), l'utilisation des prévisions climatiques est prometteuse et sans coûts, ce qui pourrait aider les agriculteurs africains à prendre des décisions stratégiques cruciales c'est-à-dire le choix des variétés, des dates de semis et utilisation des engrais qui réduiraient leur vulnérabilité et augmenteraient leur profitabilité (Ingram et al., 2002; Sultan et al., 2010; Roudier et al., 2011). Malgré l'évolution du climat, les recommandations de fertilisation n'ont cependant pas changé. Dans ce contexte, un management approprié de la fertilisation minérale en se basant sur les prévisions climatiques et/ou informations météorologiques pourrait permettre d'optimiser la productivité des

\section{MATÉRIEL ET MÉTHODES}

Sites d'étude : Deux essais ont été conduits durant la saison de pluies 2015 dans deux stations expérimentales de l'Institut Sénégalais de Recherche Agricole (ISRA) situées à Nioro du Rip $\left(13^{\circ} 44^{\prime}\right.$ latitude Nord et $15^{\circ} 46^{\prime}$ longitude Ouest) et Sinthiou Malème ( $13^{\circ} 46^{\prime}$ latitude Nord et $13^{\circ} 40^{\prime}$ longitude Ouest). Les deux localités sont caractérisées par un climat de type soudano-sahélien avec un régime pluviométrique monomodal qui s'étend de intrants en maximisant leurs effets (rendement élevé) en cas de pluviométrie favorable et en diminuant leur usage, et perte, tout en essayant d'obtenir un minimum de rendement, en cas de pluviométrie défavorable. Pour cela, il faut mieux comprendre et maîtriser les interactions entre l'azote et l'eau dans la production des cultures. En particulier, retarder et/ou réduire l'apport d'engrais du fait d'un démarrage lent ou tardif des pluies pourrait être non seulement une stratégie appropriée afin d'accroitre la production du sorgho, et donc réduire l'insécurité alimentaire mais aussi de réduire les risques de perte d'investissements sur la production. Cette étude cherche à apporter des éléments de réponse pour aborder cette question. Elle a donc pour objectif d'étudier les réponses de variétés de sorgho aux apports tardifs et/ou réduits non conventionnels de NPK et d'urée qui pourraient être dictés par les prévisions ou informations météorologiques.

juin à octobre, avec un pic en août. Les cumuls de pluies durant les expérimentations ont été de $1045 \mathrm{~mm}$ et 563 $\mathrm{mm}$ respectivement à Nioro du Rip et à Sinthiou Malème, et les températures moyennes durant les expérimentations de 28,4 et $29,1^{\circ} \mathrm{C}$ respectivement. La figure 1 montre les cumuls de pluie et les températures moyennes décadaires à Nioro du Rip et Sinthiou Malème. 


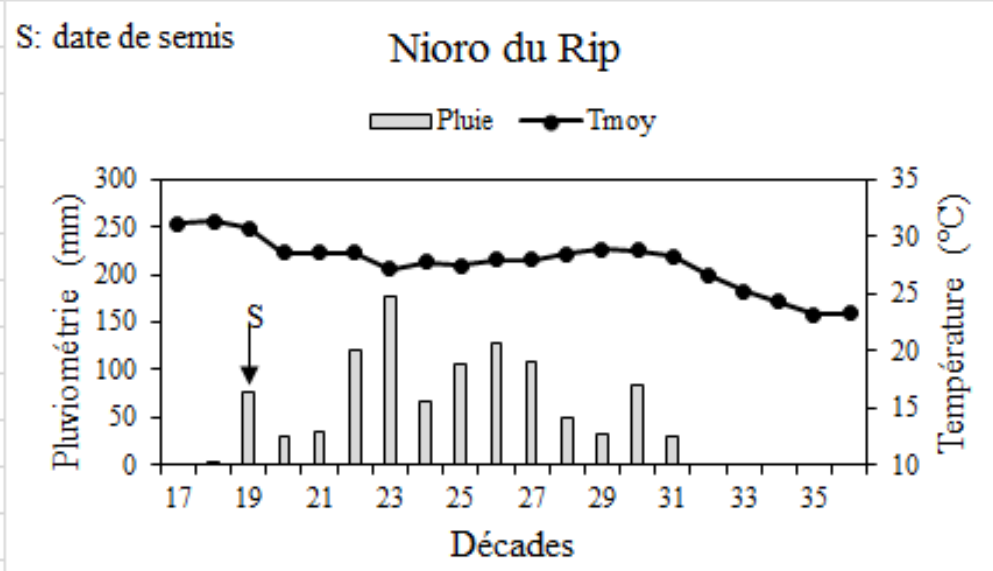

S: date de semis $\quad$ Sinthiou Malème

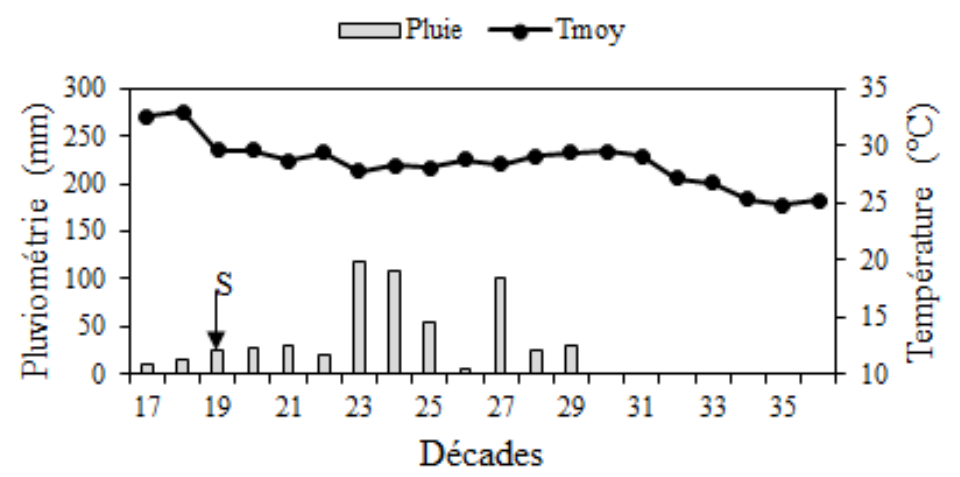

Figure 1 : Pluviométrie et température moyenne durant les expérimentations à Nioro du Rip et à Sinthiou Malème. Tmoy : Température moyenne

Les essais ont été menés sur des sols similaires, du type ferrugineux tropical lessivé, de texture sableuse et à structure fragmentaire. Des échantillons composites de sol ont été prélevés avant chaque essai pour être analysés au laboratoire de sol et végétaux du Centre National de Recherche Agronomique de Bambey au Sénégal. Les résultats d'analyses sont présentés dans le tableau 1. De ces analyses, il ressort que le sol est acide sur les deux sites $(\mathrm{pH}<6)$; les taux d'azote, du phosphore assimilable et de matière organique sont faibles dans l'ensemble mais légèrement plus importants à Nioro du Rip. Le précédent cultural sur les deux sites était de l'arachide.

Tableau 1: Caractéristiques physico-chimiques du sol avant l'expérimentation dans l'horizon 0-30 cm.

\begin{tabular}{l|l|l|l|l|l|l|l|l}
\hline Sites & $\begin{array}{l}\text { Horizon } \\
(\mathbf{c m})\end{array}$ & $\begin{array}{l}\text { Argile } \\
(\%)\end{array}$ & $\begin{array}{l}\text { Limon } \\
(\%)\end{array}$ & Sable (\%) & $\mathbf{p H}$ & $\begin{array}{l}\text { Phosphore } \\
\text { assimilable } \\
(\mathbf{p p m})\end{array}$ & $\begin{array}{l}\text { Azote } \\
\text { total (\%) }\end{array}$ & $\begin{array}{l}\text { Matière } \\
\text { organique } \\
(\%)\end{array}$ \\
\hline Nioro du & $0-10$ & 1.45 & 3.43 & 95.12 & 5.78 & 8.686 & 0.052 & 1.10 \\
Rip & $0-20$ & 5.23 & 3.72 & 91.06 & 5.17 & 4.685 & 0.038 & 1.00 \\
& $20-30$ & 4.49 & 2.82 & 92.69 & 5.68 & 5.264 & 0.065 & 1.06 \\
\hline Sinthiou & $0-10$ & 2.71 & 4.21 & 93.08 & 5.69 & 2.968 & 0.040 & 0.71 \\
Malème & $0-20$ & 2.63 & 4.15 & 93.22 & 5.29 & 2.785 & 0.035 & 0.64 \\
& $20-30$ & 2.94 & 3.46 & 93.60 & 5.51 & 2.511 & 0.037 & 0.62 \\
\hline
\end{tabular}



accroitre la production du sorgho en zones soudano-sahéliennes du Sénégal

Matériel végétal : Quatre (4) variétés améliorées de sorgho dont un hybride ont été utilisées dans cette étude (Tableau 2). Elles ont été choisies du fait de leurs caractéristiques contrastées en termes de phénologie (cycle court, moyen et long), architecture (petite et grande taille), réponse à la fertilisation (hybride réagit mieux) et usage (grain et double usage : fourrage et grain).

Tableau 2 : Caractéristiques des quatre variétés de sorgho utilisées

\begin{tabular}{l|l|l|l|l|l|l}
\hline Nom & Origine & Race & Cycle & $\begin{array}{l}\text { Sensibilité à la } \\
\text { Photopériode }\end{array}$ & Architecture & Usage \\
\hline Fadda & Mali & $\begin{array}{l}\text { Guinea- } \\
\text { hybride } \\
\text { Caudatum }\end{array}$ & Moyen & Sensible & Grande taille & $\begin{array}{l}\text { Double } \\
\text { Usage } \\
\text { Faourou }\end{array}$ \\
$\begin{array}{l}\text { Soumalemba } \\
\text { Soumba }\end{array}$ & $\begin{array}{l}\text { Sénégal } \\
\text { Caméroun }\end{array}$ & $\begin{array}{l}\text { Guinea } \\
\text { Caudatum }\end{array}$ & $\begin{array}{l}\text { Long } \\
\text { Moyen }\end{array}$ & $\begin{array}{l}\text { Sensible } \\
\text { Légèrement } \\
\text { Sensible }\end{array}$ & $\begin{array}{l}\text { Petite taille } \\
\text { Grande taille } \\
\text { Petite taille }\end{array}$ & $\begin{array}{l}\text { Grain } \\
\text { Grain } \\
\text { Double } \\
\text { usage }\end{array}$ \\
\hline
\end{tabular}

Cycle : court : de 85 to 95 jours, Moyen : de 105 to 135 jours Long : $>135$ jours

Architecture : Grande taille : $>3 \mathrm{~m}$, Petite taille $<2 \mathrm{~m}$

Dispositif expérimental et conduite de l'essai : Le dispositif expérimental sur les deux sites était un split plot à quatre répétitions. Les expérimentations sur ce plan ne différaient que par la randomisation des traitements. Les traitements étaient au nombre de 20, résultant des combinaisons des quatre (4) variétés de sorgho avec cinq (5) modalités de fertilisation différentes en termes de dose et période d'application, qui sont décrites cidessous:

(i) le "zéro", contrôle (T1, pas d'application d'engrais i.e. $0 \%)$;

(ii) $150 \mathrm{~kg} / \mathrm{ha}$ de NPK (15-15-15) à la levée $+50 \mathrm{~kg} / \mathrm{ha}$ d'urée (46\%) au tallage + $50 \mathrm{~kg} / \mathrm{ha}$ d'urée à la montaison, pratique conventionnelle et recommandée au Sénégal pour le sorgho (T2 i.e. 100\% dose, application conventionnelle) ;

(iii) $75 \mathrm{~kg} / \mathrm{ha}$ de NPK (15-15-15) à la levée $+25 \mathrm{~kg} / \mathrm{ha}$ d'urée $(46 \%)$ au tallage $+25 \mathrm{Kg} / \mathrm{ha}$ d'urée à la montaison (T3, moitié dose de T2 appliquée aux mêmes stades i.e. dose $50 \%$ ) ;

(iv) $150 \mathrm{~kg} / \mathrm{ha}$ de NPK + $50 \mathrm{~kg} / \mathrm{ha}$ d'urée à la montaison et $+50 \mathrm{~kg} / \mathrm{ha}$ d'urée à l'épiaison ( $\mathrm{T} 4$, dose $\mathrm{T} 2$ décalée, i.e. dose $100 \%$ décalée) ;

et (v) $75 \mathrm{~kg} / \mathrm{ha}$ de NPK + $25 \mathrm{~kg} / \mathrm{ha}$ d'urée à la montaison + $25 \mathrm{~kg} / \mathrm{ha}$ d'urée à l'épiaison (T5, moitié dose de T4 apportée aux mêmes stades i.e. dose $50 \%$ décalée).

Excepté la modalité T2 qui est la pratique recommandée en termes de dose et période d'apport, les autres modalités ont été déterminées en considérant des scénarii possibles d'usage et application de fertilisants découlant des prévisions climatiques et/ou pluviométries observées en début de campagne. Ainsi des modalités de type T4 et T5 pourraient être pratiquées dans l'hypothèse (h1) où les prévisions sont incertaines mais où finalement la saison démarre normalement et semble être bonne : dans ce cas on n'apporte rien au départ, puis on fait des apports tardifs. Pour des raisons de logistique il n'y a pas eu de modalité testée correspondant à l'hypothèse contraire (h2) où la saison deviendrait catastrophique après un bon démarrage, ce qui conduirait les agriculteurs à ne pas appliquer de l'urée après avoir fait un apport initial de NPK. Cependant, on peut considérer que cette situation est globalement encadrée par les modalités T1 et T3. Les traitements de fertilisation étaient assignés en parcelles principales. Les quatre variétés de sorgho étaient assignées en parcelles élémentaires. Des diguettes ont été construites autour des parcelles principales afin d'éviter des ruissellements d'engrais entre parcelles. Chaque parcelle élémentaire était constituée de 5 lignes de 6,8 m espacées de 0,8 m l'une de l'autre; l'espace inter-poquets était de $0,4 \mathrm{~m}$. Cinq (5) à six (6) grains par poquet ont été semés et démariés à deux plants par poquets à la levée donnant une densité de 62 500 plants à l'hectare. Les engrais ont été appliqués à différents stades de développement de chaque variété. Les essais ont été conduits en conditions pluviales sur les deux sites. Avant l'installation de chaque essai, un labour croisé de $30 \mathrm{~cm}$ de profondeur a été fait. Les opérations culturales (sarclage, buttage et traitements phytosanitaires) ont été réalisées régulièrement et au besoin pour maintenir les conditions optimales de culture. Collecte de données: Paramètre de croissance : la biomasse végétative (feuilles + tiges) a été collectée avant la fertilisation tardive (au tallage, B1) et après la fertilisation tardive (pleine floraison, B2). Sur chaque parcelle élémentaire, 4 poquets ont été choisis aléatoirement sur les 3 lignes centrales pour être prélevés. La matière sèche a été évaluée après séchage 
sous serre pendant 1 mois puis à l'étuve à $65^{\circ} \mathrm{C}$ (jusqu'à l'obtention de poids constant) et pesée. La matière sèche obtenue a été corrigée à $14 \%$ et rapportée à l'hectare.

Rendements grain et biomasse : à maturité 12 poquets des 3 lignes centrales ont été récoltées sur chaque parcelle élémentaire pour évaluer le rendement grain $(R G)$ et la biomasse végétative (RB) après le même processus de séchage et correction du poids à $14 \%$.

L'indice de récolte $(I R)$ et un indice de réponse à la fertilisation (RF) ont été calculés suivants les formules :

$I R_{i j}=R G_{i j} /\left(R B_{i j}+R G_{i j}\right)$

$R F_{i j}=R G_{i j} / T 2_{j}$

Où $R G_{i j}$ est le rendement grain du traitement i de la variété $j, R B_{i j}$ est le rendement en biomasse végétative $d u$

\section{RÉSULTATS}

Conditions climatiques: La température moyenne journalière a été légèrement plus élevée à Sinthiou Malème qu'à Nioro du Rip. Le site de Nioro du Rip a été plus arrosé $(1045 \mathrm{~mm})$ que celui de Sinthiou Malème $(563 \mathrm{~mm})$ et la saison a démarré plus lentement à Sinthiou Malème (Figure 1). Du fait de leur différence de cycle, les différentes variétés ont reçu des quantités de pluie variables : ainsi, Fadda a reçu en moyenne 33\% (42 et $23 \%$ ), Faourou $36 \%$ (48 et 23\%) ; Soumalemba $47 \%$ (71 et 23\%) et Soumba 33\% (42 et 23\%) du cumul de pluies avant les apports tardifs d'engrais (Figure 3). traitement i de la variété j et T2j est le rendement du traitement $\mathrm{T} 2$ de la variété $\mathrm{j}$

Analyses statistiques: Le logiciel $\mathrm{R}$ version 3.4 .0 (http://www.R-project.org/; R Development Core Team, 2017) a servi à faire des analyses de variance (ANOVA) selon le modèle en Blocs Complets Randomisés (BCR) avec combinaison de sites de Mclntosh (1983) après avoir combiné pour chaque variable les données des deux essais afin de tester la réponse des quatre variétés de sorgho aux applications de NPK et urée à différents stades sous différentes conditions environnementales. Lorsque l'ANOVA révèle une différence significative, la comparaison des moyennes a été faite à l'aide du test de la plus petite différence significative (PPDS ou LSD) au seuil de $5 \%$.

Résumé de l'ANOVA : Le facteur " environnement » a eu un effet significatif $(P<0,05)$ sur $B 1, R B$ et $R F$ alors que la fertilisation a montré un effet significatif sur B1, RG et RB. La variété a exercé un effet significatif sur toutes les variables à l'exception de B1 et RF. Par ailleurs, l'interaction $E^{\star} F$ a montré un effet significatif sur $R G$ et $R B$. L'effet de l'interaction $E^{*} V$ a été significatif sur $B 2$, $R G$ et $R B$. L'interaction $F^{*} V$ n'a été significative que sur $R G$. La triple interaction $E^{\star} F^{*} V$ n'a montré aucun effet significatif sur toutes les variables d'étude (Tableau 3).

Tableau 3 : Résumé de l'analyse de variance sur la biomasse avant (B1) et après (B2) la fertilisation tardive, rendement grain $(R G)$, biomasse $(R B)$ et indice de récolte $(I R)$ et indice de réponse à la fertilisation $(R F)$

\begin{tabular}{l|l|l|l|l|l|l}
\hline \multirow{2}{*}{ Sources } & \multicolumn{5}{l}{ Paramètres de croissance } & \multicolumn{4}{l}{ Paramètres de rendement } \\
\cline { 2 - 7 } & B1 & B2 & RG & RB & IR & RF \\
\hline Environnement $(\mathrm{E})$ & $<0,0001$ & 0,0697 & 0,8082 & 0,0483 & 0,3266 & 0,0131 \\
Fertilisation (F) & $<0,0001$ & 0,1388 & 0,0005 & 0,0004 & 0,7989 & 0,7412 \\
Variété $(\mathrm{V})$ & 0.0547 & $<0,0001$ & $<0,0001$ & $<0,0001$ & $<0,0001$ & 0,3002 \\
$\mathrm{E}^{\star} \mathrm{F}$ & 0,3477 & 0,3143 & 0,05 & 0,0073 & 0,9320 & 0,4119 \\
$\mathrm{E}^{\star} \mathrm{V}$ & 0,6997 & 0.0047 & 0,0005 & 0,0417 & 0,2192 & 0,1218 \\
$\mathrm{~F}^{\star} \mathrm{V}$ & 0,4459 & 0,3686 & 0,0176 & 0,2665 & 0,6184 & 0,8853 \\
$\mathrm{E}^{*} \mathrm{~F}^{*} \mathrm{~V}$ & 0,9854 & 0,5797 & 0,8647 & 0,4612 & 0,5022 & 0,8139 \\
\hline
\end{tabular}

B1 : Biomasse avant fertilisation tardive, B2 : Biomasse après fertilisation tardive, RG : Rendement grain, RB :

Rendement biomasse, IR : Indice de récolte, $\mathrm{RF}$ : indice de réponse à la fertilisation 
Ganyo et al., J. Appl. Biosci. 2018 Optimisation du NPK et urée basée sur les informations climatiques pour accroitre la production du sorgho en zones soudano-sahéliennes du Sénégal

Tableau 4 : Effet de l'environnement, de la fertilisation et de la variété sur la biomasse, rendement grain, indice de récolte et l'indice de réponse à la fertilisation

\begin{tabular}{|c|c|c|c|c|c|c|}
\hline \multirow[b]{2}{*}{ Sources de variation } & \multicolumn{2}{|c|}{ Paramètres de croissance } & \multicolumn{4}{|c|}{ Paramètres de rendement } \\
\hline & $\begin{array}{l}\text { B1 } \\
\text { (kg/ha) }\end{array}$ & $\begin{array}{l}\text { B2 } \\
\text { (kg/ha) }\end{array}$ & $\begin{array}{l}\text { RG } \\
\text { (kg/ha) }\end{array}$ & $\begin{array}{l}\text { RB } \\
\text { (kg/ha) }\end{array}$ & IR & RF \\
\hline \multicolumn{7}{|l|}{ Environnement $(\mathrm{E})$} \\
\hline $\begin{array}{l}\text { Nioro du Rip } \\
\text { Sinthiou Malème }\end{array}$ & $\begin{array}{l}96 a \\
15 b\end{array}$ & $\begin{array}{l}5361 \\
6315\end{array}$ & $\begin{array}{l}915 \\
931\end{array}$ & $\begin{array}{l}5044 a \\
4289 b\end{array}$ & $\begin{array}{l}0,20 \\
0,23\end{array}$ & $\begin{array}{l}0,78 \mathrm{~b} \\
208 \mathrm{a}\end{array}$ \\
\hline \multicolumn{7}{|l|}{ Fertilisation (F) } \\
\hline T1 & $62 \mathrm{~b}$ & 4813 & $671 d$ & $3524 \mathrm{~b}$ & 0,19 & 1,14 \\
\hline $\mathrm{T} 2$ & $105 a$ & 6652 & 1233 a & $5566 \mathrm{a}$ & 0,20 & 1 \\
\hline T3 & $110 \mathrm{a}$ & 5690 & $962 b c$ & $4989 a$ & 0,22 & 1,36 \\
\hline T4 & $65 b$ & 6298 & $1007 a b$ & $5561 \mathrm{a}$ & 0,24 & 1,27 \\
\hline T5 & $58 b$ & 5270 & $746 \mathrm{~cd}$ & $3722 \mathrm{~b}$ & 0,22 & 2,09 \\
\hline \multicolumn{7}{|l|}{ Variétés (V) } \\
\hline Fadda & 69 & 7793 a & $1265 \mathrm{a}$ & $6184 b$ & $0,22 b$ & 0,76 \\
\hline Faourou & 69 & $3718 c$ & $911 \mathrm{~b}$ & $1834 d$ & $0,36 \mathrm{a}$ & 1,38 \\
\hline Soumalemba & 93 & $5545 b$ & $758 \mathrm{~b}$ & $7304 a$ & $0,20 \mathrm{~b}$ & 1,15 \\
\hline Soumba & 90 & $5655 b$ & $752 b$ & $3278 c$ & $0,09 \mathrm{c}$ & 2,17 \\
\hline \multicolumn{7}{|l|}{ Interactions } \\
\hline$E^{*} F$ & ns & ns & * & ** & ns & ns \\
\hline$E^{*} V$ & ns & ** & ** & * & ns & ns \\
\hline$F^{*} \mathrm{~V}$ & ns & ns & * & ns & ns & ns \\
\hline$E^{*} F^{*} V$ & ns & ns & ns & ns & ns & ns \\
\hline
\end{tabular}

Les valeurs dans une colonne suivies de la même lettre ne sont pas significativement différentes au seuil de $5 \%$. B1: Biomasse avant fertilisation tardive, B2 : Biomasse après fertilisation tardive, RG : Rendement grain, RB : Rendement biomasse, IR : Indice de récolte, $\mathrm{RF}:$ Réponse à la fertilisation ; T1 = pas d'engrais ; T2 = $150 \mathrm{~kg} / \mathrm{ha}$ de NPK (15-15-15) à la levée $+50 \mathrm{~kg} / \mathrm{ha}$ d'urée (46\%) au tallage + $50 \mathrm{Kg} / \mathrm{ha}$ d'urée à la montaison ; T3 $=75 \mathrm{~kg} / \mathrm{ha}$ de NPK (15-15-15) à la levée + $25 \mathrm{~kg} / \mathrm{ha}$ d'urée (46\%) au tallage + $25 \mathrm{Kg} / \mathrm{ha}$ d'urée à la montaison; $\mathrm{T} 4=150 \mathrm{~kg} / \mathrm{ha}$ de NPK $+50 \mathrm{~kg} / \mathrm{ha}$ d'urée à la montaison $+50 \mathrm{~kg} / \mathrm{ha}$ d'urée à l'épiaison; $\mathrm{T} 5=75$ $\mathrm{kg} / \mathrm{ha}$ de NPK (15-15-15) $+25 \mathrm{~kg} / \mathrm{ha}$ of urée $(46 \%)$ à la montaison $+25 \mathrm{Kg} / \mathrm{ha}$ d'urée à l'épiaison ; ns : non significatif, " significatif à $\mathrm{P}$ $<0.05,{ }^{* *}$ significatif à $P<0.01$

Évolution de la biomasse durant le cycle : Le tableau 4 présente les valeurs moyennes de biomasses (avant et après fertilisation tardive et à la récolte), le rendement grain et les indices de récolte et de réponse à la fertilisation par environnement, fertilisation et variété. La B1 a été significativement plus importante à Nioro du Rip qu'à Sinthiou Malème. Les plantes avant l'application tardive d'engrais étaient stressées avec des productions de biomasse significativement moins importantes sur les traitements tardifs (T4 et T5) que sur les traitements avec le timing conventionnel (T2 et T3). En effet, la biomasse sous T4 était inférieure à celle sous $\mathrm{T} 2$ et celle sous T5 aussi inférieure à T3. Après la fertilisation tardive, aucun effet significatif de la fertilisation sur la biomasse n'a été observé. On en déduit, que les plants sous T4 et T5 ont recouvert leur développement normal avec des biomasses statistiquement équivalentes à celles sous $\mathrm{T} 2$ et $\mathrm{T} 3$ lorsqu'on les compare deux à deux $(P=0.1388)$.
Par ailleurs, la biomasse après la fertilisation tardive a varié en fonction des variétés : Fadda $(7793 \mathrm{~kg} / \mathrm{ha})$ a donné significativement plus de biomasse que Soumalemba et Soumba (respectivement $5545 \mathrm{~kg} / \mathrm{ha}$ et $5655 \mathrm{~kg} / \mathrm{ha}$ ) ; Faourou ayant produit la plus faible quantité de biomasse à cette date.

Effet de l'environnement, de la fertilisation et de la variété sur les rendements et les indices IR et RF

Rendement grain : Les rendements suivant l'interaction $E^{\star} \mathrm{F}$ (en combinant les variétés) ont varié de 582 à 1390 $\mathrm{kg} / \mathrm{ha}$ à Nioro du Rip et de 739 à $1065 \mathrm{~kg} / \mathrm{ha}$ à Sinthiou Malème. A Nioro du Rip, c'est la dose $100 \%$ appliquée au timing recommandé qui a induit le rendement le plus élevé (1390 kg/ha) suivie de la moitié dose (T3, 1039 $\mathrm{kg} / \mathrm{ha}$ ) et de la dose $100 \%$ appliquée tardivement (T4, $948 \mathrm{~kg} / \mathrm{ha}$ ). L'application tardive de $50 \%$ d'engrais (T5) a donné un rendement $(583 \mathrm{~kg} / \mathrm{ha})$ équivalent au témoin 0 $\%$ d'engrais $(\mathrm{T} 1,615 \mathrm{~kg} / \mathrm{ha})$. Par contre, à Sinthiou 
Malème, les applications de $100 \%$ de la dose d'engrais recommandée, en timing conventionnel (T2) et en décalé (T4) ont induit les rendements les plus élevés, respectivement 1039 et $1065 \mathrm{~kg} / \mathrm{ha}$, suivies de la moitié dose en timing conventionnel (T3) et en décalé (T5) et du témoin T1. Les rendements issus des apports tardifs en particulier ceux de T5 ont été meilleurs à Sinthiou Malème qu'à Nioro du Rip (Figure 2). Comparant la pratique recommandée ( $\mathrm{T} 2$, dose et période d'application) à la pratique introduite ( $\mathrm{T} 4$, apport tardif de la même dose), excepté Soumba qui a moins bien réagi à la fertilisation tardive sur les deux sites, l'apport de la dose $100 \%$ en décalé a donné des performances au-dessus du rendement moyen de sorgho au Sénégal de 2012 à 2016 (888 kg/ha) lorsque le cumul de pluies avant la fertilisation tardive (stade montaison) est inférieur à 50\% du cumul d'eau durant la période de culture et/ou des performances supérieures au rendement de T2 lorsque ce dernier est inférieur à la moyenne nationale de 2012 à 2016 (Figure 3). Ainsi, à Nioro du Rip et Sinthiou Malème, l'apport décalé de $100 \%$ d'engrais dans la saison a donné des niveaux de rendements satisfaisants. La figure 4 illustre le rendement grain des 4 variétés de sorgho en fonction des modalités de fertilisation. Chez Fadda et
Soumba, l'application de la dose $100 \%$ selon le timing recommandé (T2) a donné les meilleures performances (1883 kg/ha et $1174 \mathrm{~kg} / \mathrm{ha}$ ) suivie de T3 et T4. Pour Faourou, la modalité $T 2$ a donné le rendement maximum mais qui est statistiquement équivalents à ceux de T3, T4 et T5. Chez Soumalemba, ce sont les apports tardifs d'engrais (T4 et T5) qui ont induit des rendements maximums (1022 et $919 \mathrm{~kg} / \mathrm{ha}$ ), mais aucune différence significative n'a été observée entre eux. Les apports de 100 et $50 \%$ de la dose recommandée dans le timing conventionnel ont donné les faibles performances pour cette variété. T4 et T5 de Soumalemba présentent une augmentation respectivement de $88 \%$ et $69 \%$ par rapport à la recommandation (T2). Les quatre variétés ont donc répondu différemment à la fertilisation. Fadda a été la variété qui a répondu au mieux à la fertilisation. Les résultats des interactions $E^{*} F$ et $F^{*} V$ (Figures 2 et 4 ) ont montré aussi que les apports tardifs en particulier de la dose $100 \%$, se révèlent bénéfiques par rapport au témoin sans apport d'engrais qui est la pratique paysanne couramment observée de nos jours du fait des incertitudes pluviométriques même si de façon surprenante certains résultats du témoin restent élevés. 


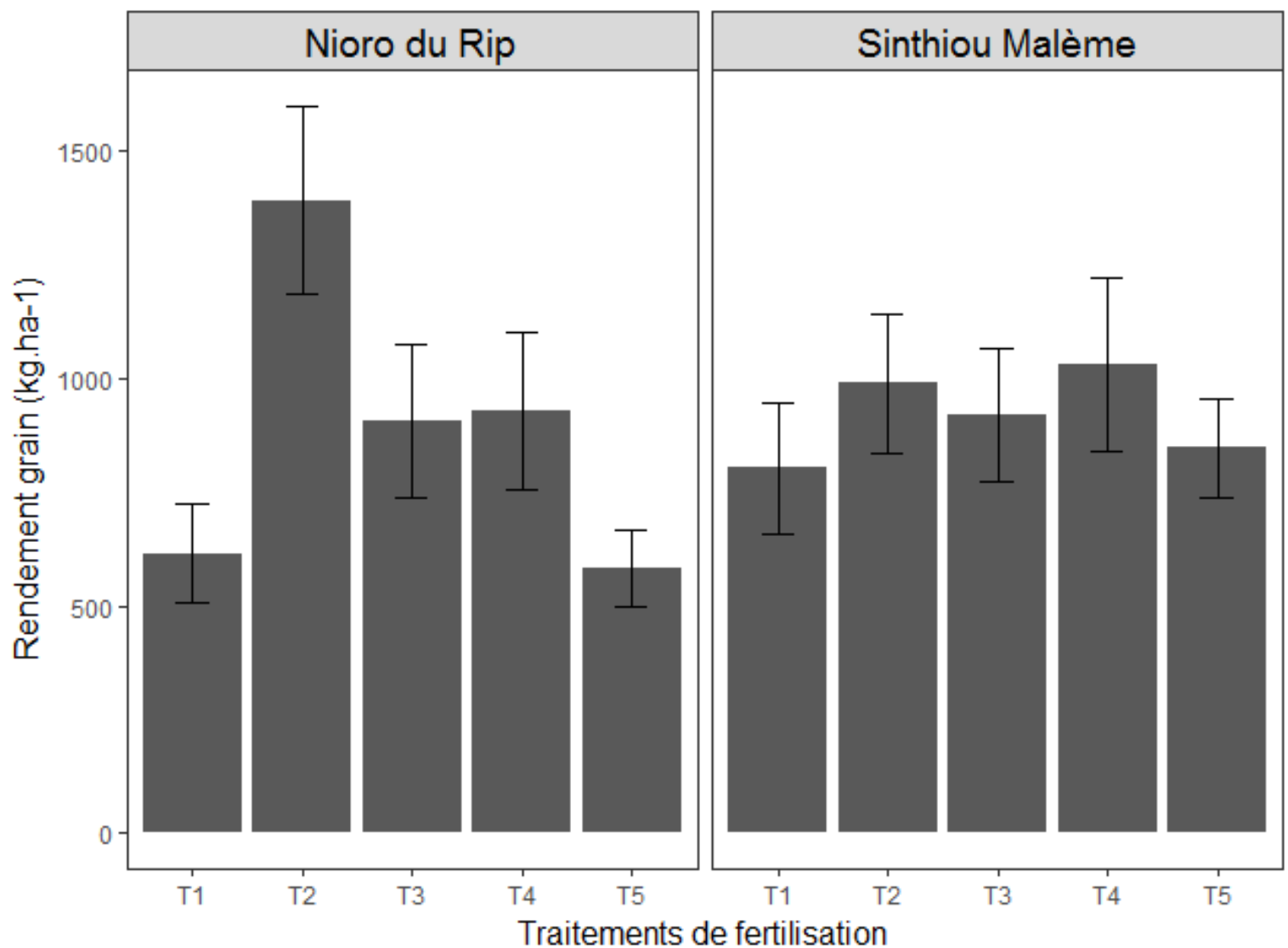

Figure 2 : Rendement grain en réponse à l'interaction environnement-fertilisation.

$\mathrm{T} 1=$ pas d'engrais ; T2 = $150 \mathrm{~kg} / \mathrm{ha}$ de NPK (15-15-15) à la levée + $50 \mathrm{~kg} / \mathrm{h}$ a d'urée $(46 \%)$ au tallage + $50 \mathrm{Kg} / \mathrm{ha} \mathrm{d'urée} \mathrm{à}$ la montaison ; T3 $=75 \mathrm{~kg} / \mathrm{ha}$ de NPK (15-15-15) à la levée $+25 \mathrm{~kg} / \mathrm{ha}$ d'urée (46\%) au tallage $+25 \mathrm{Kg} / \mathrm{ha}$ d'urée à la montaison; $T 4=150 \mathrm{~kg} / \mathrm{h}$ de NPK $+50 \mathrm{~kg} / \mathrm{ha}$ d'urée à la montaison $+50 \mathrm{~kg} / \mathrm{ha}$ d'urée à l'épiaison; $T 5=75 \mathrm{~kg} / \mathrm{ha} \mathrm{de}$ NPK (15-15-15) + $25 \mathrm{~kg} / \mathrm{ha}$ of urée $(46 \%)$ à la montaison $+25 \mathrm{Kg} / \mathrm{ha}$ d'urée à l'épiaison 

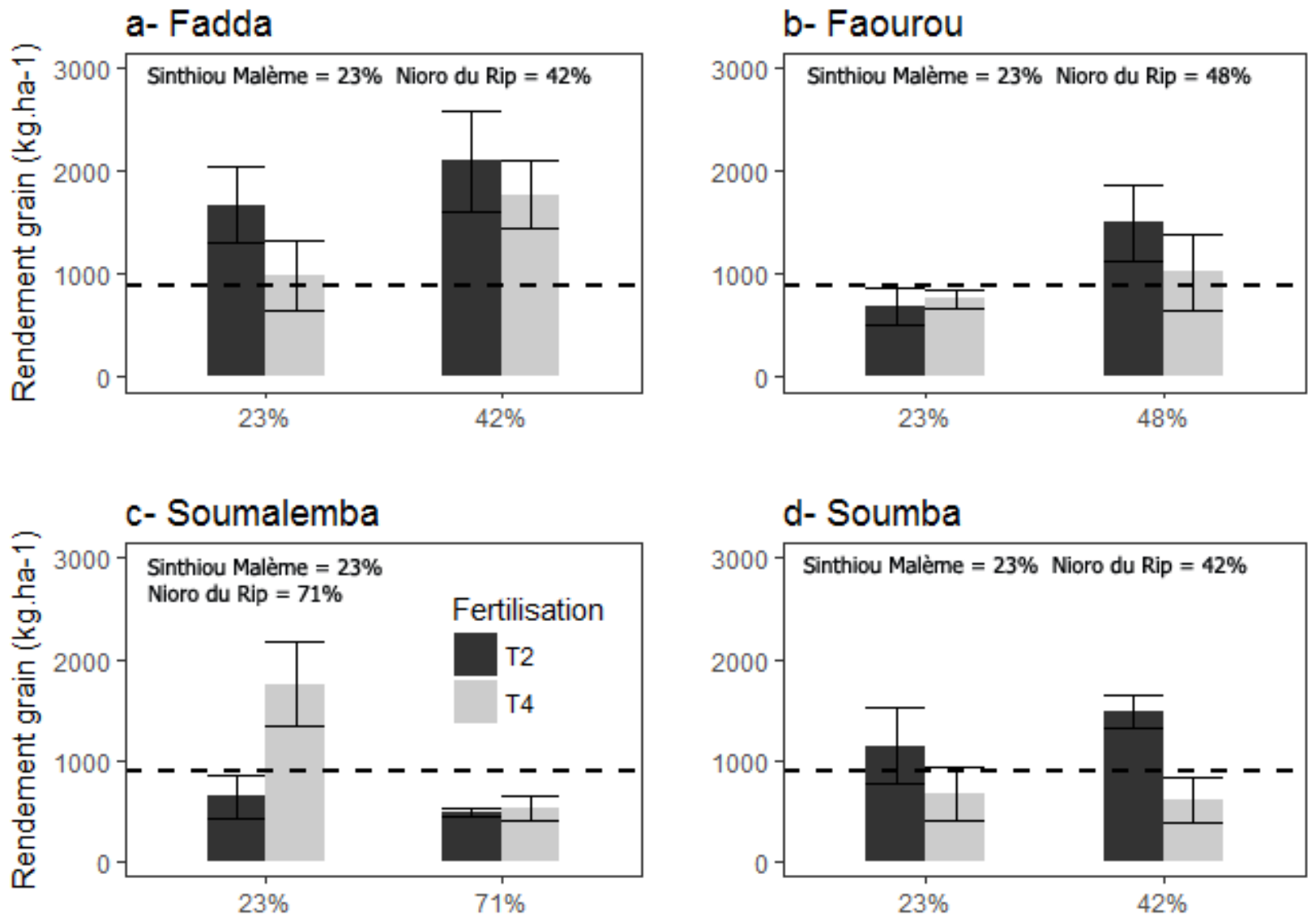

Eau reçue avant la fertilisation tardive

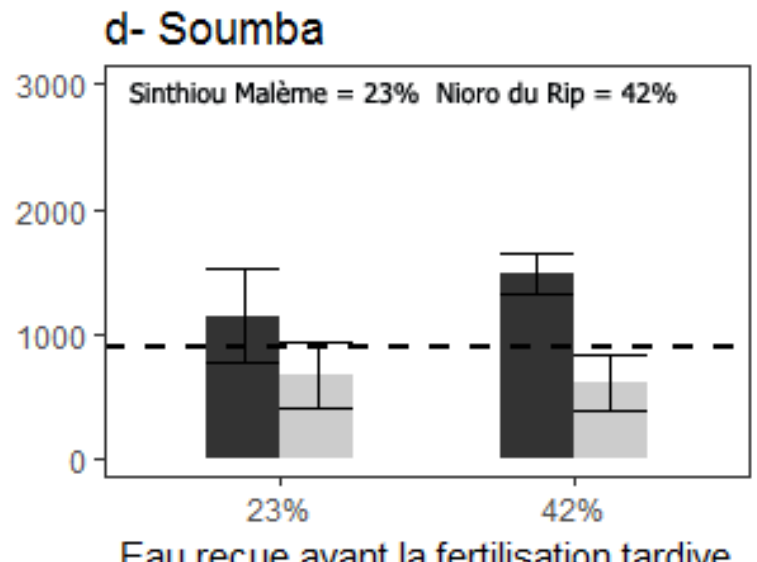

Figure 3 : Comparaison du rendement grain en fonction de la quantité de pluie (en pourcentage) reçue avant les applications tardives d'engrais

$\mathrm{T} 2=150 \mathrm{~kg} / \mathrm{ha}$ de NPK (15-15-15) à la levée $+50 \mathrm{~kg} / \mathrm{ha}$ d'urée (46\%) au tallage + $50 \mathrm{Kg} / \mathrm{ha}$ d'urée à la montaison T4 = $150 \mathrm{~kg} / \mathrm{ha}$ de NPK + $50 \mathrm{~kg} / \mathrm{ha}$ d'urée à la montaison + $50 \mathrm{~kg} / \mathrm{ha}$ d'urée à l'épiaison 

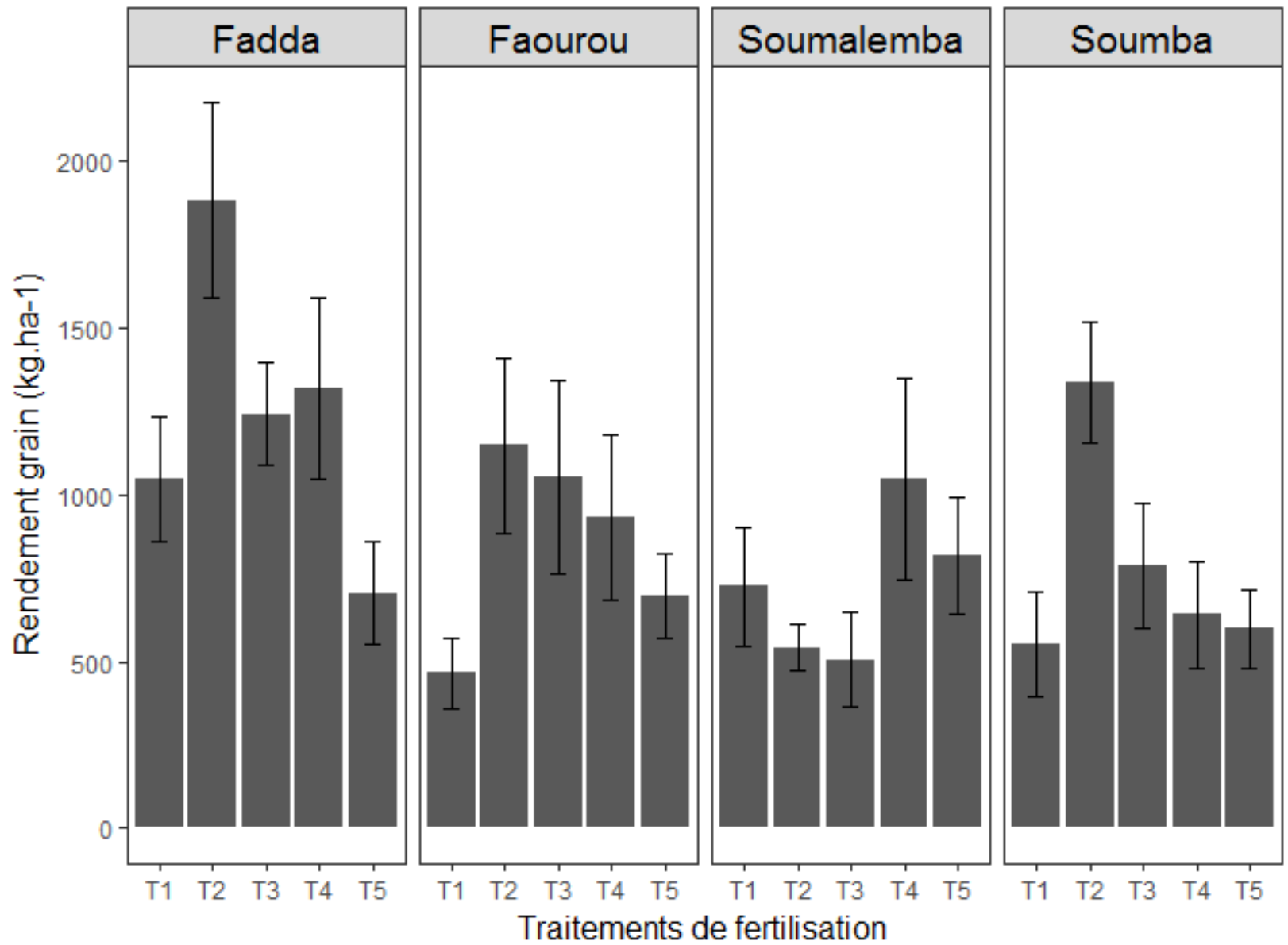

Figure 4 : Rendement grain des variétés de sorgho en fonction des modalités de fertilisation.

$\mathrm{T} 1=$ pas d'engrais ; T2 $=150 \mathrm{~kg} / \mathrm{ha}$ de NPK (15-15-15) à la levée $+50 \mathrm{~kg} / \mathrm{ha}$ d'urée $(46 \%)$ au tallage $+50 \mathrm{Kg} / \mathrm{ha}$ d'urée à la montaison ; T3 $=75 \mathrm{~kg} / \mathrm{ha}$ de NPK (15-15-15) à la levée $+25 \mathrm{~kg} / \mathrm{ha} \mathrm{d'urée} \mathrm{(46 \% )} \mathrm{au} \mathrm{tallage}+25 \mathrm{Kg} / \mathrm{ha}$ d'urée à la montaison ; T4 = $150 \mathrm{~kg} / \mathrm{ha}$ de NPK $+50 \mathrm{~kg} / \mathrm{ha}$ d'urée à la montaison $+50 \mathrm{~kg} / \mathrm{ha}$ d'urée à l'épiaison ; T5 $=75 \mathrm{~kg} / \mathrm{ha}$ de NPK $(15-15-15)+25 \mathrm{~kg} / \mathrm{ha}$ of urée $(46 \%)$ à la montaison $+25 \mathrm{Kg} / \mathrm{ha}$ d'urée à l'épiaison

Rendement biomasse : La biomasse végétative à la récolte a été plus importante à Nioro du Rip qu'à Sinthiou Malème. La variété Soumalemba a donné un rendement paille significativement plus élevé suivie de Fadda, Soumba et Faourou (Tableau 4). La figure 5 montre les valeurs du rendement paille en fonction des modalités de fertilisation sur chaque site. II en ressort qu'à Nioro du Rip, les apports $100 \%$ (T2 et T4) et l'apport $50 \%$ en timing conventionnel (T3) ont induit des niveaux de biomasses supérieurs (différence significative) à ceux des deux autres traitements ( $\mathrm{T} 1$ et T5). Cependant, à Sinthiou Malème les rendements paille de tous les traitements avec apport d'engrais et le témoin sans apports sont statistiquement identiques entre eux. Les apports tardifs de $100 \%$ d'engrais ont donnés des rendements de paille plus élevés que sans apports d'engrais (Figure 5). 


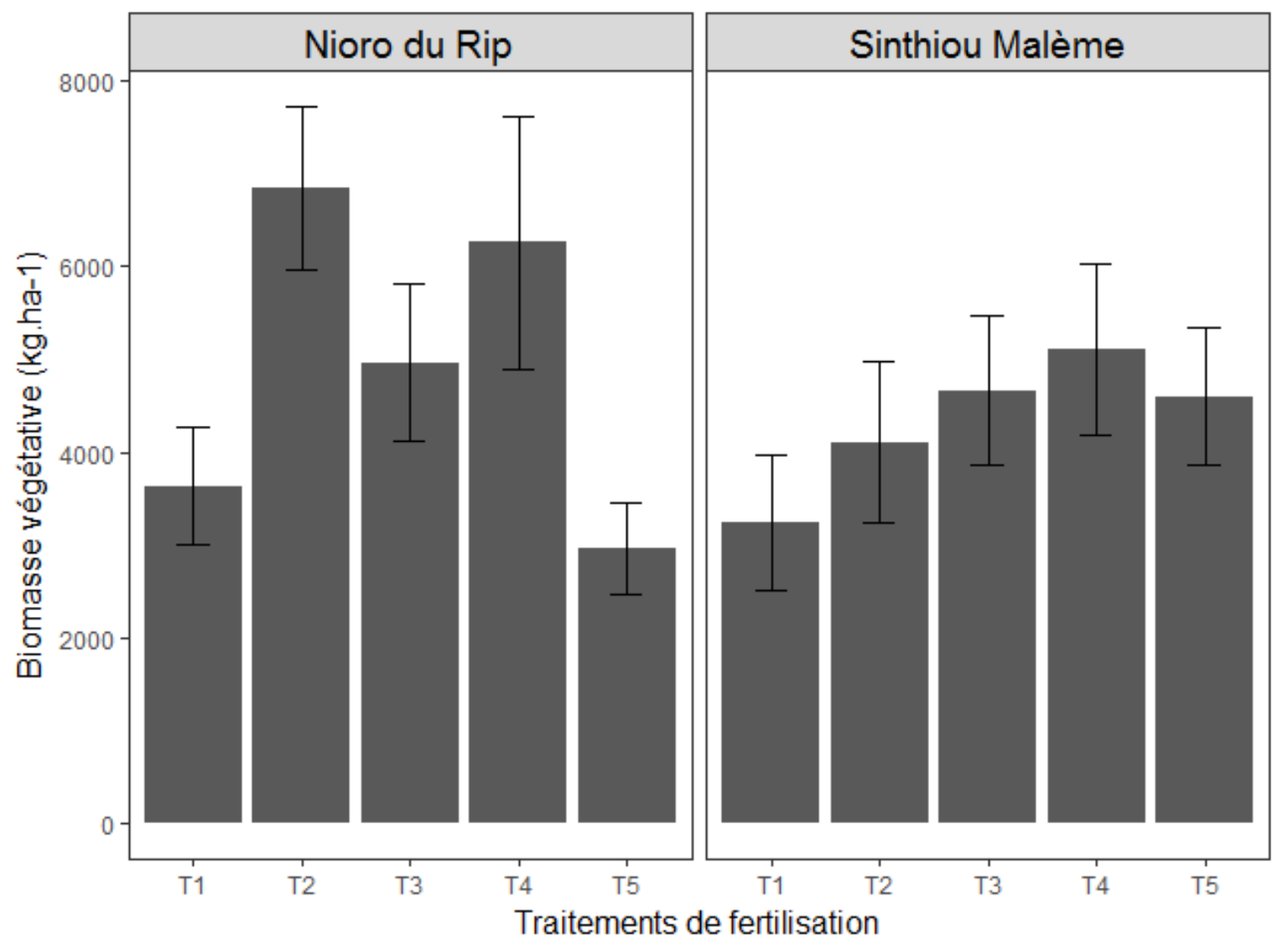

Figure 5 : Rendement en biomasse affecté par l'interaction environnement-fertilisation.

$\mathrm{T} 1=$ pas d'engrais ; T2 = $150 \mathrm{~kg} / \mathrm{ha}$ de NPK (15-15-15) à la levée $+50 \mathrm{~kg} / \mathrm{ha} \mathrm{d'urée} \mathrm{(46 \% )} \mathrm{au} \mathrm{tallage}+50 \mathrm{Kg} / \mathrm{ha}$ d'urée à la montaison ; T3 $=75 \mathrm{~kg} / \mathrm{ha}$ de NPK (15-15-15) à la levée $+25 \mathrm{~kg} / \mathrm{ha} \mathrm{d'urée} \mathrm{(46 \% )} \mathrm{au} \mathrm{tallage}+25 \mathrm{Kg} / \mathrm{ha}$ d'urée à la montaison ; $\mathrm{T} 4=150 \mathrm{~kg} / \mathrm{ha}$ de NPK + $50 \mathrm{~kg} / \mathrm{ha}$ d'urée à la montaison $+50 \mathrm{~kg} / \mathrm{ha}$ d'urée à l'épiaison ; T5 = $75 \mathrm{~kg} / \mathrm{ha}$ de NPK (15-15-15) + 25 $\mathrm{kg} / \mathrm{ha}$ of urée $(46 \%)$ à la montaison $+25 \mathrm{Kg} / \mathrm{ha}$ d'urée à l'épiaison

Indices : Seul le facteur variété a eu un effet significatif sur l'indice de récolte. Ainsi, la variété Faourou a donné le maximum du ratio grain-biomasse aérienne totale qui est supérieur à ceux de Fadda et Soumalemba ; eux-mêmes supérieurs à celui de Soumba; alors que l'indice de réponse à la fertilisation $(\mathrm{RF})$, a varié seulement en

\section{DISCUSSION}

Les résultats de cette étude ont permis d'évaluer les réponses de quatre variétés de sorgho à des stratégies contrastées d'apports d'engrais minéraux (NPK et urée). Ainsi, en considérant le paramètre de développement qu'est la biomasse, l'apport tardif d'engrais a permis aux plantes de sorgho initialement affectées par le non-apport d'engrais au départ, de recouvrir un développement normal avec des quantités de matière sèche similaires entre les apports conventionnels et décalés (Tableau 4). Ce regain de croissance montre que même avec un début de saison difficile ou catastrophique, un apport fonction de l'environnement avec la meilleure réponse à Sinthiou Malème. Bien que la différence n'ait pas été significative pour la fertilisation, les stratégies définies montrent cependant des indices numériquement plus élevés que celui de $\mathrm{T} 2$.

tardif des engrais peut permettre de booster la croissance des plants. Les plantes peuvent donc utiliser et bénéficier de ces apports d'engrais. Ce regain de croissance pourrait être attribué d'une part à l'apport de nutriments spécialement de l'azote qui aurait affecté la croissance des plantes par la division cellulaire et l'agrandissement des cellules (Stals et Inzé 2001). D'autre part, l'humidité du sol induit par l'eau reçue après la fertilisation tardive peut affecter l'expression des macro-éléments apportés aux plantes et vice versa. Ce qui aurait stimulé le développement par une bonification de l'effet de l'Azote 

accroitre la production du sorgho en zones soudano-sahéliennes du Sénégal

(N). L'effet de l'interaction $E^{*} F$ n'est pas significatif sur la biomasse avant et après la fertilisation tardive. Cependant, cette interaction est significative sur la paille à la récolte. Selon Mon et al. (2016), la fertilisation azotée affecte significativement la biomasse aérienne à partir d'un seuil d'humidité. Ce qui pourrait expliquer les effets observés de cette interaction aux différentes dates de prélèvement. Le rendement grain a varié en fonction de l'effet interactif de l'environnement (eau reçue) et de la fertilisation ; en particulier les apports tardifs d'engrais ont varié en fonction des sites (Figure 2). L'augmentation du rendement grain due à l'application tardive n'est pas constante au fil des années et des localités (Woolfolk et al., 2002 ;Blandino et al., 2015), ce qui est conforme à nos résultats. Le lessivage des nutriments dû à la quantité et fortes intensités des pluies reçues à Nioro du Rip pourrait expliquer les moins bonnes performances de rendement à ce site. En effet, sur ce site nous avions enregistré de grosses pluies allant de 75 à plus de 100 $\mathrm{mm}$ par jour, ce qui serait mauvais pour les plantes sur un sol sableux avec un fort lessivage. Par ailleurs, les bonnes performances obtenues à partir des apports tardifs ont été rapportés sur le sorgho en zones semiarides d'Iran (Amiri et al., 2014), sur le riz dans les plaines de Philippines (Perez et al., 1996) et sur le blé d'hiver en pots (Xue et al., 2016). En effet, Amiri et al. (2014) rapportent des rendements de sorgho plus élevés avec l'apport $25 \%$ de $\mathrm{N}$ au semis, $50 \%$ de $\mathrm{N}$ à la montaison et $25 \%$ de $\mathrm{N}$ à la floraison. Cependant, les résultats sur les bonnes performances des traitements " tardifs » contrastent avec ceux obtenus sur le riz par Fageria et Baligar (1999). Ces auteurs ont obtenu des niveaux de rendements bas avec deux traitements de fertilisation incluant des apports tardifs à l'épiaison et à la floraison définis comme (i) un tiers de $\mathrm{N}$ apporté au semis, un tiers de $\mathrm{N}$ à l'initiation paniculaire et un tiers de $\mathrm{N}$ à floraison et (ii) un apport de zéro $\mathrm{N}$ au semis, un tiers

\section{CONCLUSION}

L'apport tardif d'engrais NPK et urée a eu un effet significatif sur la croissance des plantes. Initialement stressées, les plantes ont recouvert le développement normal comme si les apports étaient faits tôt dans la saison. La période d'apport des engrais combinée à la distribution de l'eau a eu un effet sur les rendements. La stratégie de fertilisation tardive a été bénéfique avec l'apport de la dose complète qui a donné des rendements de $\mathrm{N}$ à l'initiation paniculaire, un tiers de $\mathrm{N}$ à l'épiaison et un tiers de $\mathrm{N}$ à la floraison. Par ailleurs, la réponse des quatre variétés à la fertilisation a été variable; Fadda ayant répondu mieux à la fertilisation en générale, Soumalemba mieux à la fertilisation tardive (Figure 4) et Soumba avec des rendements liés à la fertilisation tardive toujours faibles par rapport à l'application conventionnelle (T2) (Figure 3). Cette variation de réponse à la fertilisation à travers des rendements différents peut être attribuée à un effet combiné du caractère variétal (i.e. caractéristique génétique) et de l'environnement (climat, sol). Ces résultats sont similaires à ceux de Blandino et al. (2015) qui ont rapporté des rendements variables et contrastés de deux variétés de blé à la fertilisation azotée tardive. Aussi, Kante et al. (2017) ont-ils rapporté des rendements variables de sorgho (hybrides et accessions locales de sorgho) au Mali. L'indice de récolte n'a varié qu'en fonction des variétés. Ce qui démontre qu'il est lié au caractère génétique du matériel végétal utilisé. La nonsignificativité de la fertilisation sur la RF traduit le potentiel de la stratégie de fertilisation tardive et/ou réduite. Finney et al., 1957 ont montré que des apports en particulier de l'azote entre l'après tallage et l'épiaison augmentent le rendement du blé. Les interactions $E^{\star} F^{*} V$ n'ont pas été significatives pour toutes les variables étudiées signifient que les variétés de sorgho se sont comportées de la même manière par rapport à la fertilisation dans les deux environnements. La stratégie d'apporter des engrais notamment $100 \%$ de la dose recommandée en fonction des prévisions météorologiques ou déroulement de la saison a permis d'obtenir des rendements au-dessus de la moyenne nationale et de la pratique paysanne courante (pas d'apport d'engrais). La fertilisation tardive se révèle être une des stratégies pour maximiser ou optimiser les rendements de sorgho en zones SoudanoSahélienne du Sénégal.

au-dessus de la pratique paysanne (pas d'apport d'engrais) et supérieurs lorsque la quantité d'eau reçue avant la fertilisation tardive est moins de $50 \%$ des cumuls reçus toute la période de culture et un faible potentiel du site pour T2. La réponse à la fertilisation notamment tardive des variétés de sorgho a été variable. Ajuster la fertilisation sur le régime pluviométrique pourrait permettre d'optimiser la productivité du sorgho. 
Ganyo et al., J. Appl. Biosci. 2018 Optimisation du NPK et urée basée sur les informations climatiques pour accroitre la production du sorgho en zones soudano-sahéliennes du Sénégal

\section{REMERCIEMENTS}

Les auteurs remercient le Programme de Productivité de l'Agriculture en Afrique de l'Ouest-Sénégal (PPAAOSénégal) qui a financé ce travail. Les auteurs sont aussi

\section{REFERENCES BIBLIOGRAPHIQUES}

Amadou, M., Gandah, M., Bielders, C.L., van Duivenbooden, N., 1999. Optimizing soil water use in Niger: research, development, and perspectives, in: van Duivenbooden, N., Pala, M., Studer, C., Bielders, C.L. (Eds.), Efficient Soil Water Use: The Key to Sustainable Crop Production in Dry Areas of West Asia, and North and Sub-Saharan Africa. Niamey, Niger 26 - 30 April 1998 and Oman, Jordan 9 - 13 May 1998. ICARDA and ICRISAT, Aleppo, Syria, pp. 143164.

Amiri, M., Mojaddam, M., Shokouhfar, A., Bakhtiarinejad, N., 2014. The effect of different levels and time of nitrogen application on grain yield, some physiological traits and nitrogen use effiency in grain sorghum. Indian J. Fundam. Appl. Life Sci. 4, 223-227.

Blandino, M., Vaccino, P., Reyneri, A., 2015. Late-season nitrogen increases improver common and durum wheat quality. Agron. J. 107, 680-690. https://doi.org/10.2134/agronj14.0405

Challinor, A., Wheeler, T., Garforth, C., Craufurd, P., Kassam, A., 2007. Assessing the vulnerability of food crop systems in Africa to climate change. Clim. Change 83, 381-399.

Fageria, N.K., Baligar, V.C., 1999. Yield and yield components of lowland rice as influenced by timing of nitrogen fertilization. J. Plant Nutr. 22, 23-32.

https://doi.org/10.1080/01904169909365603

Finney, K.F., Meyer, J.W., Smith, F.W., Fryer, H.C., 1957. Effect of foliar spraying of Pawnee wheat with urea solutions on yield, protein content and protein quality. Agron. J. 49, 341-347. https://doi.org/10.2134/agronj1957.0002196200 4900070001x

Gerstenmier, A., Choho, T., 2015. Fourniture d'intrants agricoles. Dakar (Sénégal).

Gueye, T., Sine, B., Cisse, N., Diatta, C., Ndiaye, S., 2016. Characterization of Phenotypic Diversity of Sorghum Collection for Developing Breeding Material. Int. J. Sci. 5, 38-48. https://doi.org/10.18483/ijSci.931

Hansen, J.W., 2002. Realizing the potential benefits of climate prediction to agriculture: issues, reconnaissants à tous les techniciens et étudiants du CERAAS qui ont participé à la collecte des données.

approaches, challenges. Agric. Syst. 74, 309330. https://doi.org/10.1016/S0308$521 \times(02) 00043-4$

Hulme, M., Doherty, R., Ngara, T., New, M., Lister, D., 2001. African climate change: $1900-2100$. Clim. Res. 17, 145-168. https://doi.org/10.3354/cr017145

Ingram, K.T., Roncoli, M.C., Kirshen, P.H., 2002. Opportunities and constraints for farmers of west Africa to use seasonal precipitation forecasts with Burkina Faso as a case study. Agric. Syst. 74, 331-349. https://doi.org/10.1016/S0308-521X(02)00044-6

IPCC, 2007. Climate change 2007: Impacts, Adaptations and Vulnerability. Contribution of Working Group II to the Fourth Assessment of the Intergovernmental Panel on Climate Change. Cambridge, UK.

Kante, M., Rattunde, H.F.W., Leiser, W.L., Nebié, B., Diallo, B., Diallo, A., Touré, A.O., Weltzien, E., Haussmann, B.I.G., 2017. Can tall guinea-race sorghum hybrids deliver yield advantage to smallholder farmers in West and Central Africa? Crop Sci. 57, 833-842. https://doi.org/10.2135/cropsci2016.09.0765

McIntosh, M.S., 1983. Analysis of combined experiments. Agron. J. 75, 153-155. https://doi.org/10.2134/agronj1983.0002196200 $7500010041 \mathrm{x}$

Mon, J., Bronson, K.F., Hunsaker, D.J., Thorp, K.R., White, J.W., French, A.N., 2016. Interactive effects of nitrogen fertilization and irrigation on grain yield, canopy temperature, and nitrogen use efficiency in overhead sprinkler-irrigated durum wheat. F. Crop. Res. 191, 54-65. https://doi.org/10.1016/j.fcr.2016.02.011

Perez, C.M., Juliano, B., Liboon, S.P., Alcantara, J.M., Cassman, K.G., 1996. Effects of late nitrogen fertilizer application on head rice yield, protein content, and grain quality of rice. Cereal Chem. $73,556-560$.

R Development Core Team, 2017. R: A language and Environment for statistical computing. R Foundation for Statistical Computing. Vienna, Austria. 
Roudier, P., Sultan, B., Quirion, P., Baron, C., Alhassane, A., Traoré, S.B., Muller, B., 2011. An ex-ante evaluation of the use of seasonal climate forecasts for millet growers in SW Niger. Int. J. Climatol. 32, 759-771. https://doi.org/10.1002/joc.2308

Slingo, J.M., Challinor, A.J., Hoskins, B.J., Wheeler, T.R., 2005. Introduction: food crops in a changing climate. Philos. Trans. R. Soc. B 360, 19831989. https://doi.org/10.1098/rstb.2005.1755

Stals, H., Inzé, D., 2001. When plant cells decide to divide. Trends Plant Sci. 6, 359-364. https://doi.org/10.1016/S1360-1385(01)02016-7

Sultan, B., Barbier, B., Fortilus, J., Mbaye, S.M., Leclerc, G., 2010. Estimating the potential economic value of seasonal forecasts in West Africa: A long-term Ex-Ante assessment in Senegal. Am. Meteorol. Soc. 2, 69-87. https://doi.org/10.1175/2009WCAS1022.1

Vanlauwe, B., Bationo, A., Chianu, J., Giller, K.E., Merckx, R., Mokwunye, U., Ohiokpehai, O., Pypers, P., Tabo, R., Shepherd, K.D., Smaling, E.M.A., Woomer, P.L., Sanginga, N., 2010. Integrated soil fertility management: Operational definition and consequences for implementation and dissemination. Outlook Agric. 39, 17-24. https://doi.org/10.5367/000000010791169998

Woolfolk, C.W., Raun, W.R., Johnson, G. V., Thomason, W.E., Mullen, R.W., Wynn, K.J., Freeman, K.W., 2002. Influence of late-season foliar nitrogen application on yield and grain nitrogen in winter wheat. Agron. J. 94, 429-434.

Xue, C., Erley, S.A.G., Rossmann, A., Schuster, R., Koehler, P., Mühling, K.-H., 2016. Split nitrogen application improves wheat baking quality by influencing protein composition rather than concentration. Front. Plant Sci. 7, 1-11. https://doi.org/10.3389/fpls.2016. 